\title{
Spectral Clustering Algorithms for Ultrasound Image Segmentation
}

\author{
Neculai Archip ${ }^{1}$, Robert Rohling ${ }^{2}$, Peter Cooperberg ${ }^{3}$, Hamid Tahmasebpour ${ }^{3}$, \\ and Simon K. Warfield ${ }^{1}$ \\ 1 Computational Radiology Laboratory, Harvard Medical School, Departments of \\ Radiology, Brigham and Women's Hospital, Children's Hospital, Boston, MA, USA \\ narchip@bwh.harvard.edu \\ 2 Department of Electrical and Computer Engineering, University of British \\ Columbia, Vancouver, BC, Canada \\ 3 Department of Radiology, University of British Columbia, Vancouver, BC, Canada
}

\begin{abstract}
Image segmentation algorithms derived from spectral clustering analysis rely on the eigenvectors of the Laplacian of a weighted graph obtained from the image. The NCut criterion was previously used for image segmentation in supervised manner. We derive a new strategy for unsupervised image segmentation. This article describes an initial investigation to determine the suitability of such segmentation techniques for ultrasound images. The extension of the NCut technique to the unsupervised clustering is first described. The novel segmentation algorithm is then performed on simulated ultrasound images. Tests are also performed on abdominal and fetal images with the segmentation results compared to manual segmentation. Comparisons with the classical NCut algorithm are also presented. Finally, segmentation results on other types of medical images are shown.
\end{abstract}

\section{Introduction}

Successful segmentation techniques can be used in a variety of clinical applications. In clinical practise, ultrasound images are often segmented manually, but manual techniques can be laborious. More sophisticated techniques are needed. Various methods have been previously proposed. For instance, active contours have been used [1]. Kalman filters are proposed in [2] to improve the segmentation of organ cavities in ultrasound. A statistical shape model is reported for aiding prostate segmentation 3 .

However, most of these methods require intensive human interaction. Spectral methods for data clustering have received increasing attention for practical applications in other fields 4. The spectral methods usually involve taking the top eigenvectors of a matrix constructed from a similarity metric (such as the distance between points) and using them to cluster the various points [5]. Image segmentation can be described as a clustering problem where pixels are the measured data points. For example, Shi and Malik [6] combine spectral clustering and image segmentation. Nevertheless, two important issues need to be 
addressed within this context. First is the determination of the number of clusters to be obtained (supervised vs unsupervised clustering). The second aspect to be considered is execution time. For real images, the matrix for which the eigenvectors should be computed is large $\left(n^{2} \times n^{2}\right.$ for an image of $n \times n$ pixels). Therefore efficiency is necessary for practical implementations. We focus on the first issue, introducing an unsupervised algorithm. Moreover, using a simple windowing principle, the introduced algorithm has reasonable execution times.

In this paper we show the effectiveness of this novel approach for ultrasound image segmentation. We present validation results on both simulated ultrasound and patient data. Manual segmentation of images by two independent radiologists is the gold standard used to quantify the success of our method. Comparison with a classical related segmentation method - NCut - is also provided. Additionally, we present examples of segmentation results from other imaging modalities.

\section{Method}

The NCut technique can be applied directly to the ultrasound image, but good results in MRI are reported for a combination of NCut with an anisotropic diffusion filter [7]. Therefore, an anisotropic filter is first applied.

\section{Spectral Clustering and Some Previous Results on Image Segmenta-}

tion. Spectral methods for image segmentation use the eigenvectors and eigenvalues of a matrix derived from the pairwise affinities of pixels. These eigenvectors induce an embedding of the pixels in a low-dimensional subspace wherein a simple central clustering method can be used to do the final partitioning. Here the partitioning is based on the Normalized Cut (NCut) 6]. Some mathematical notations are first introduced. Let $I$ be the original image of size $N \times N$. The symmetric matrix $S \in R^{N^{2} \times N^{2}}$ denotes the weighted adjacency matrix for a graph $G=(V, E)$ with nodes $V$ representing pixels and edges $E$ whose weights capture the pairwise affinities between pixels. Let $A$ and $B$ represent a bipartition of $V$, i.e. $A \cup B=V$ and $A \cap B=\emptyset$. Let $\operatorname{cut}(A, B)$ denote the sum of the weights between $A$ and $B: \operatorname{cut}(A, B)=\sum_{i \in A, j \in B} S_{i j}$. The degree of the $i^{t h}$ node is defined as $d_{i}=\sum_{j} S_{i j}$. The total connection from nodes in the set $A$ to all nodes in the graph is denoted by $\operatorname{assoc}(A, V)=\sum_{i \in A, j \in V} S_{i j}$. The normalized cut between sets $A$ and $B$ is then given by:

$$
N C u t(A, B)=\frac{\operatorname{cut}(A, B)}{\operatorname{assoc}(A, V)}+\frac{\operatorname{cut}(A, B)}{\operatorname{assoc}(B, V)} \text {. }
$$

The problem is to find $A$ and $B$ such that $\operatorname{NCut}(A, B)$ is minimized. Using elements of spectral graph theory, it is shown [6] that an approximate solution may be obtained by thresholding the eigenvector (called the Fiedler vector) corresponding to the second eigenvalue $\lambda_{2}$ of the generalized eigenvalue problem: $(D-S) y=\lambda D y$, where $D$ is a diagonal matrix with entries $D_{i i}=d_{i}$. Image segmentation is reduced to the problem of partitioning the set $V$ into disjoint 
sets $V_{1}, . ., V_{n}$, such that similarity among nodes in $V_{i}$ is high and similarity across $V_{i}$ and $V_{j}$ is low. Here we define the similarity function between two pixels $i$ and $j$ as:

$$
S_{i j}=\left\{\begin{array}{lll}
\frac{1-\frac{\|F(i)-F(j)\|_{2}}{\max F-\operatorname{minF} F}}{\|X(i)-X(j)\|_{2}} & \text { if } & \|X(i)-X(j)\|_{2}<r \\
0 & \text { otherwise }
\end{array}\right\}
$$

where $X(i)$ is the spatial location of node $i$, and $F(i)$ is a feature vector, based on intensity. The values $\max F$ and $\min F$ are respectively the maximum and the minimum values of $F$ for the all the pixels in the image. Finally, the similarity matrix $S$ is normalized by $S=S / \max (S)$ as proposed by [8]. A requirement of NCut is the number of clusters. Overcoming this problem is a challenging task. Unsupervised clustering, based solely on Fiedler eigenvector is a potential solution. In the unsupervised clustering, the user does not need to explicitly specify the number of clusters. More details follow.

Fiedler Eigenvector for Unsupervised Image Segmentation. Instead of minimizing the NCut function (as suggested by [6]), a property of the Fiedler vector is used. Let each pixel be $I_{j}, j=1, \ldots, N^{2}$. Given the image pixels $P=$ $\left(I_{1}, . ., I_{N^{2}}\right)$ and the Fiedler vector $V=\left(v_{1}, . ., v_{N^{2}}\right)$, we consider the permutation $\wp=\left(i_{1}, . ., i_{N^{2}}\right)$ that sorts the vector $V: v_{i_{1}} \leq . . \leq v_{i_{N^{2}}}$. By applying $\wp$ to the pixel vector $P$, the vector $\left(I_{i_{1}}, . ., I_{i_{N^{2}}}\right)$ is obtained. It satisfies the property that $\exists j_{1}, . ., j_{k}$ with $\forall p, j_{p} \leq l \leq j_{p+1}: S\left(I_{l}, I_{l-1}\right)<\epsilon$, where $S$ is the similarity function defined in equation (2). This property provides a way to cluster the original image according to the given similarity metric.

In its new form the vector components are grouped in compact blocks which represent the clusters. It becomes clear that the problem of clustering the initial matrix data is reduced to the problem of determining the sequences of maximum length having similar values in the vector obtained from the Fiedler eigenvector. The pseudocode of the introduced unsupervised segmentation technique is listed in Algorithm 1 .

Calculating the eigenvectors for the Laplacian resulting from the whole image is computationally expensive. Therefore a grid is used to divide the whole image into smaller windows. We then apply the clustering algorithm to each of these smaller windows. A global criterion is used to regroup the cluster obtained in every grid cell. There is still no need to know or estimate the number of clusters, $K$, in advance.

The introduced algorithm requires three parameters to be specified. The first parameter (DifPixels) specifies the maximum difference between two pixels to consider them as belonging to the same cluster. This parameter could be determined for specific applications using a priori information about the image content. However, for our experiments we used the same value for all examples with good results. The second parameter (DifClusters) is the threshold value when two clusters are grouped together during windowing. It can also be customized for a particular application, but is kept constant in the images presented in this paper. Both parameters are present in the majority of the standard segmentation algorithms since one needs to specify when two pixels are similar enough to 


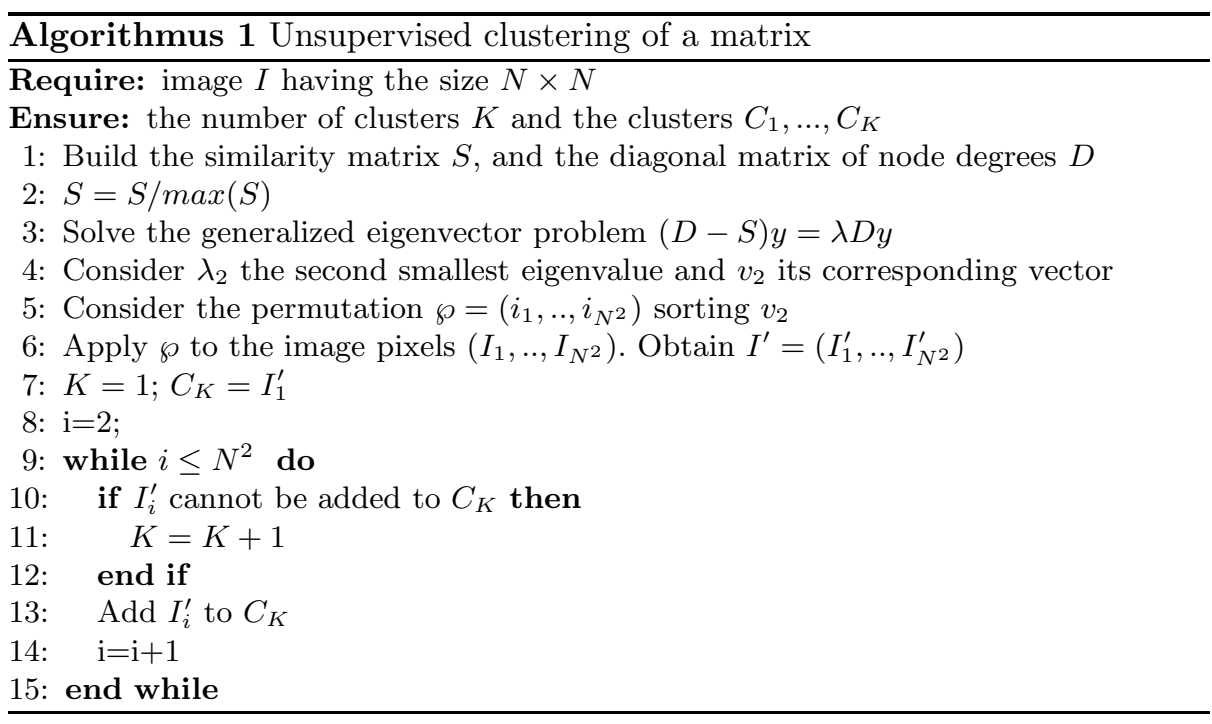

be grouped in the same region. The third parameter is the window size and is also kept constant. We have also noticed that there are small differences in the clustering results when using different values for the window size. More details follow in the Results section.

\section{Results}

Five simulated ultrasound images of circular objects were created with the Field II program 9]. Various levels of Gaussian additive noise have been used.

Two different metrics are used to compute the error between the boundary of the segmented region and the known object: Hausdorff and the mean error. Although theoretically attractive, the Hausdorff metric is very sensitive to noise in practice.

Abdominal images were also obtained in vivo for patients exhibiting cysts in the liver. The cysts have varying degrees of visibility so are more difficult to segment. Five images were used (see Fig. 1).

The clustering techniques are also used to segment the amniotic fluid present in five images of the fetus. Clinical applications of the segmentation include the diagnosis and quantification of hydramnios or oligohydramnios (see Fig. 2). An example of an ultrasound image segmentation obtained during a prostate brachytherapy procedure is shown in Fig. 3. Finally results of this technique on other imaging modalities, such as CT and microscopic images are illustrated in the Fig. 4. The Hausdorff and mean errors were calculated between the radiologist and NCut, and the radiologist and NCut-Unsupervised.

For the liver images, a second radiologist performed manual segmentation so a comparison can be made between the results with each radiologist. For all tests, 


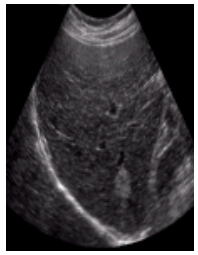

(a)

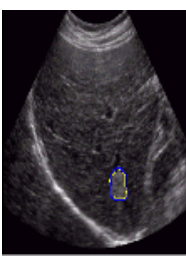

(b)

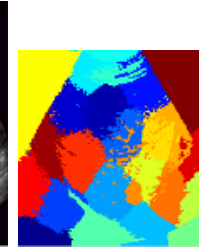

(c)

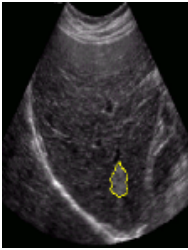

(d)

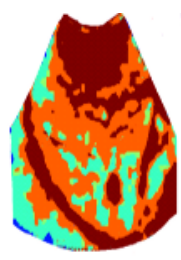

(e)

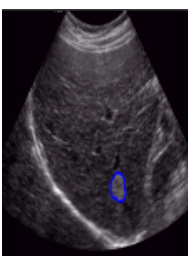

(f)

Fig. 1. Liver cyst image. (a) Original image of a liver with a visible cyst. (b) The manually drawn contour of the cyst. (c) Segmentation using NCUT (9 clusters). (d) The contour of the selected cluster corresponding to the cyst. (e) Segmentation using our unsupervised technique. (f) The contour of the selected cluster corresponding to the cyst.

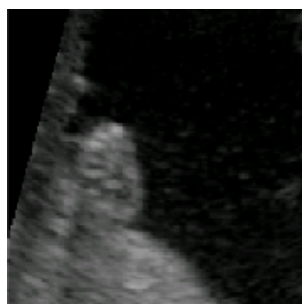

(a)

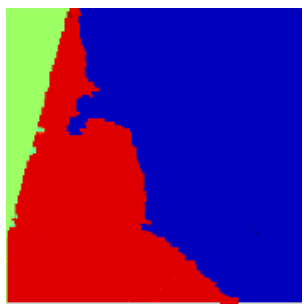

(b)

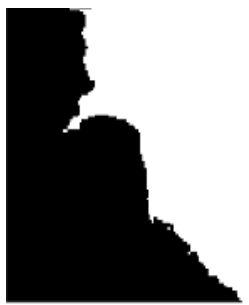

(c)

Fig. 2. Fetus images. (a) An original image of a fetus. (b) Segmentation using NCut. (c) Segmentation using our unsupervised technique. The segmentation closely reflects the underlying anatomy.

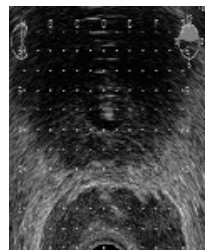

(a)

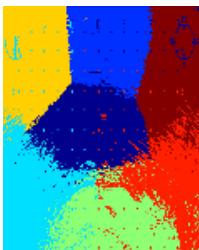

(b)

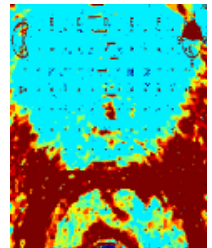

(c)

Fig. 3. Bladder image (a) Original image. (b) Segmentation with NCut. (c) Segmentation with our unsupervised method.

the NCut and NCut-Unsupervised were implemented in Matlab (The Mathworks Inc., Natick, MA, USA). For NCut, the number of clusters was selected manually for each image to get the best segmentation. For NCut-Unsupervised, there was no need to specify the number of clusters. The parameters used for the algorithm had the same values for all the images in the experiments. The values used in the tests are DifPixels $=5$, DifClusters $=20$ and window size $=15$ pixels. The computation time of the Matlab implementation of both NCut and NCut-Unsupervised is approximately 30 seconds on an image of $150 \times 150$. The results in all cases is 


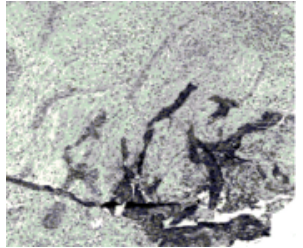

(a)

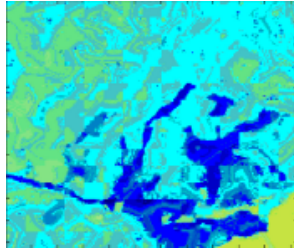

(b)

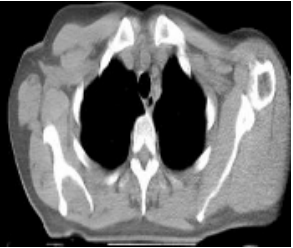

(c)

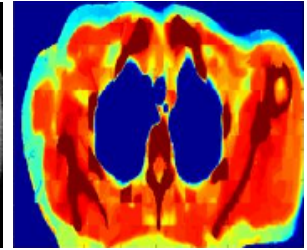

(d)

Fig. 4. (a) Original cancer stain image. (b) Segmentation with our method. (c) CT slice. (d) its segmentation. The segmentation closely reflects the underlying anatomy.

Table 1. Simulated ultrasound images. The errors between the segmented object and the true object are calculated using the Hausdorff metric and the mean differences.

\begin{tabular}{|c|c|c|c|c|}
\hline Image & \begin{tabular}{c} 
True object vs NCut \\
\cline { 2 - 5 }
\end{tabular} $\begin{array}{c}\text { Trusdorff } \\
(\mathrm{mm})\end{array}$ & $\begin{array}{c}\text { Mean } \\
(\mathrm{mm})\end{array}$ & $\begin{array}{c}\text { Hausdorff } \\
(\mathrm{mm})\end{array}$ & $\begin{array}{c}\text { Mean } \\
(\mathrm{mm})\end{array}$ \\
\hline 1 & 3.5 & 2.2 & 4.0 & 2.0 \\
\hline 2 & 3.6 & 1.3 & 3.8 & 1.5 \\
\hline 3 & 3.7 & 1.2 & 3.2 & 1.4 \\
\hline 4 & 3.5 & 1.4 & 3.3 & 1.2 \\
\hline 5 & 2.7 & 1.9 & 2.2 & 1.3 \\
\hline Average & $\mathbf{3 . 4}$ & $\mathbf{1 . 6}$ & $\mathbf{3 . 3}$ & $\mathbf{1 . 5}$ \\
\hline
\end{tabular}

Table 2. Liver cyst images. The errors between NCut-Unsupervised and a manually segmented contour of the cyst are calculated using the Hausdorff metric and the mean differences. The manually segmented contours were drawn by radiologist Rad.1 or Rad.2

\begin{tabular}{|c|c|c|c|c|}
\hline Image & $\begin{array}{c}\text { Hausdorff Error } \\
\text { Rad.1 vs NCut-Uns. } \\
(\mathrm{mm})\end{array}$ & $\begin{array}{c}\text { Mean Error } \\
\text { Rad.1 vs NCut-Uns. } \\
(\mathrm{mm})\end{array}$ & $\begin{array}{c}\text { Hausdorff Error } \\
\text { Rad.2 vs NCut-Uns. } \\
(\mathrm{mm})\end{array}$ & $\begin{array}{c}\text { Mean Error } \\
\text { Rad.2 vs NCut-Uns. } \\
(\mathrm{mm})\end{array}$ \\
\hline 1 & 6.5 & 2.6 & 6.0 & 2.4 \\
\hline 2 & 2.3 & 1.2 & 2.0 & 1.1 \\
\hline 3 & 1.7 & 0.8 & 2.5 & 1.1 \\
\hline 4 & 2.5 & 1.2 & 2.4 & 1.0 \\
\hline 5 & 1.2 & 0.5 & 1.6 & 0.7 \\
\hline Average & $\mathbf{2 . 8}$ & $\mathbf{1 . 3}$ & $\mathbf{2 . 9}$ & $\mathbf{1 . 3}$ \\
\hline
\end{tabular}

a partitioning of the image into a set of regions. The clinical user then can select the segmented region of interest and subsequently calculate geometric properties (dimensions, shape, area, volume), build anatomical models, or other types of analysis. For this work, the boundaries of the segmented regions are compared. Numerical results are presented in the Tables 11 , 2 and 3. Performance estimates for the segmentation of liver cyst image presented in the Fig. 1 were also obtained using STAPLE [10]. Overall accuracy of the segmentation, summarized as the probability for any voxel that the true segmentation label matched the rater segmentation, was 0.981 (Fig. 1 (b)), 0.965 (Fig. 11 (d)) and 0.989 (Fig. 1 (e)). 
Table 3. Fetus images. The errors between NCut, NCut-Unsupervised and a manually segmented contour of the amniotic fluid are calculated using the Hausdorff metric and the mean differences.

\begin{tabular}{|c|c|c|c|c|}
\hline \multirow{2}{*}{ Image } & \multicolumn{2}{|c|}{ Manual vs NCut } & Manual vs NCut-Unsupervised \\
\cline { 2 - 5 } & Hausdorff & Mean & Hausdorff & Mean \\
\hline 1 & 6.4 & 2.8 & 6.0 & 2.2 \\
\hline 2 & 5.5 & 2.9 & 5.7 & 3.2 \\
\hline 3 & 5.1 & 3.2 & 3.8 & 3.1 \\
\hline 4 & 5.5 & 3.0 & 5.2 & 2.9 \\
\hline 5 & 4.5 & 2.2 & 4.7 & 1.7 \\
\hline Average & $\mathbf{5 . 4}$ & $\mathbf{2 . 8}$ & $\mathbf{5 . 3}$ & $\mathbf{2 . 6}$ \\
\hline
\end{tabular}

\section{Discussion}

The simulations show a good match between the spectral clusters and the true object. Small differences along the boundary are likely due to the noise and speckle in the images. There is no significant difference between the NCut and the NCut-Unsupervised techniques (at the $95 \%$ confidence level using the paired student t-test). This gives confidence that the changes to the algorithm to make it unsupervised have not caused a drop in accuracy. For the tests on the liver cysts, there was also no significant difference between the following: 1. errors between NCut and manual segmentation and 2. the errors between the two manual segmentations (95\% confidence level, using the paired t-test). Nor was there a significant difference between the following: 1. errors between NCut-Unsupervised and manual segmentation and 2. the errors between the two manual segmentations (again with same confidence, 95\% using the paired t-test). In other words, the results from either NCut or NCut-unsupervised were within the variation of the two radiologists.

For the tests on the amniotic fluid segmentation, there was also no significant difference between the NCut and theNCut-Unsupervised techniques when compared to the manual segmentation results ( $95 \%$ confidence level, paired ttest). With NCut-Unsupervised, the fluid and the fetus were clearly partitioned into separate clusters. On the other hand, as previously mentioned, the clusters obtained with standard NCut tend to have the same size, so the amniotic fluid was occasionally split in two clusters, and manually combined by clicking both regions.

All of the tests were done without changing the three parameters of the method. The images of the partitioned regions also show the limitations of a spectral clustering approach. Clearly not all separate tissue types are partitioned into separate regions. The objects of interest in this study were partitioned the easiest because they exhibited the most homogeneous properties. For example, the liver cyst forms a single cluster, but the rest of the liver is formed from four more clusters. Some of the liver clusters are also shared with non-liver tissue. Clearly the liver could not be completely segmented with the current partition. So far, the method appears to work well for fluid-filled cavities and vessels. 
To perform other segmentation tasks, a more sophisticated similarity criterion may be needed. Additional features, such as texture could be added to the similarity criterion. This may slow the creation of the similarity matrix, but the rest of the algorithm will proceed without change. The main conclusion is that automatic unsupervised spectral clustering on ultrasound images is feasible. The spectral clustering technique is promising, and future research will focus on the development of new similarity criteria, the addition of other a priori information, and further improvements to speed.

\section{Acknowledgments}

This investigation was supported by NSF ITR 0426558, a research grant from the Whitaker Foundation, a research grant from CIMIT, by NIH grants R21 MH67054, R01 LM007861 and P41 RR13218, by Natural Sciences and Engineering Research Council of Canada and by the Institute of Robotics and Intelligent Systems.

\section{References}

1. Ladak, H., Downey, D., Steinman, D., Fenster, A.: Semi-automatic technique for segmentation of the prostate from $2 d$ ultrasound images. In: Proceedings of IEEE BMES/EMBS Conference on Serving Humanity, Advanced Technology. (1999)

2. Abolmaesumi, P., Sirouspour, M.: An interacting multiple model probabilistic data association filter for cavity boundary extraction from ultrasound images. IEEE Transactions on Medical Imaging 23 (2004) 772-784

3. Shen, D., Zhan, Y., Davatzikos, C.: Segmentation of the prostate boundaries from ultrasound images using statistical shape model. IEEE Transactions on Medical Imaging 22 (2003) 539-551

4. Kluger, Y., Basri, R., Chang, J., Gerstein, M.: Spectral biclustering of microarray data: coclustering genes and conditions. Genome Research 13 (2003) 703-716

5. Ng, A.Y., Jordan, M., Weiss, Y.: On spectral clustering: Analysis and an algorithm. In: Advances in Neural Information Processing Systems 14, MIT Press (2002) 849 856

6. Shi, J., Malik, J.: Normalized cuts and image segmentation. IEEE Transactions on Pattern Analysis and Machine Intelligence 22 (2000) 888-905

7. Carballido-Gamio, J., Belongie, S., Majumdar, S.: Normalized cuts in 3-d for spinal mri segmentation. IEEE Transactions on Medical Imaging 23 (2004)

8. Meila, M., Shi, J.: Learning segmentation by random walks. In: Neural Information Processing Systems. (2000) 873-879

9. Jensen, J.: A new calculation procedure for spatial impulse responses in ultrasound. Journal of the Acoustical Society of America 105 (1999) 3266-3274

10. Warfield, S., Zou, K., Wells, W.: Simultaneous truth and performance level estimation (staple): an algorithm for the validation of image segmentation. IEEE Transactions on Medical Imaging 23 (2004) 903-21 